\title{
Ensino do cuidado humanizado: evolução e tendências da produção científica*
}

\author{
Teaching of the humanized care: evaluation and trends of the scientific production
}

Enseñanza del cuidado humanizado: evaluación y tendencias de la producción cientifica

\section{Juliana Caldas de Souza', Juliana de Oliveira Roque e Lima', Denize Bouttelet Munari', Elizabeth Esperidiãol}

'Universidade Federal de Goiás. Departamento de Enfermagem, Núcleo de Estudos e Pesquisas em Saúde Integral. Goiânia, GO

\section{RESUMO}

Desde a divulgação das Diretrizes Curriculares de Enfermagem os cursos de graduação têm missão de formar enfermeiros generalistas, críticos, reflexivos e humanistas, o Que requer esforços das instituições formadoras na busca de estratégias Que viabilizem desenvolvimento dessas habilidades. Estudo descritivo, exploratório de natureza bibliográfica, teve objetivo de analisar a produção cientifica em periódicos nacionais sobre ensino do cuidado humanizado na graduação em enfermagem entre 1990/2008. A ocorrência dessa produção nos periódicos estudados sinaliza tendência de expansão do ensino do cuidado humanizado na formação do enfermeiro. Na análise identificamos a contribuição das Diretrizes Curriculares, além de estratégias facilitadoras no ensino do cuidado humanizado. Para o desenvolvimento dessa competência é fundamental ênfase nessa dimensão na formação acadêmica e a educação permanente.

Descritores: Educação em enfermagem; Estudantes de enfermagem; Humanização da assistência; Recursos humanos em saúde. and humanist nurse, what requests efforts of the education institutions in the search of strategies that make possible the development of those abilities. Descriptive study of exploratory of bibliographical nature, had as objective to analyze scientific production in nationals periodic about teaching of the humanized care on the graduation in nursing, among 1990/2008. The occurrence of the production the studied newspapers us allows to affirm that there is a tendency each time bigger about the nurse formation in what he concerns the teaching of the humanized care. In the analysis we identified the Curricular Directories contribution, besides facilitative strategies in the teaching of the humanized care. For the development of that competence it is fundamental emphasis in that dimension in the academic formation and the permanent education.

Descriptors: Education, nursing; Students, nursing; Humanization of the attendance; Health manpower.

\section{RESUMEN}

Desde la divulgación de los Directorios Curriculares de Enfermería los cursos de graduación tienen la misión de formar el enfermero generalista, crítico, reflexivo y humanista, lo que pide esfuerzos de las instituciones de educación en la búsqueda de estrategias Que hacen posible el desarrollo de esas habilidades. La ocurrencia de esta en los periódicos estudiados señaliza una tendencia de expansión de la enseñanza del cuidado humanizado en la formación del enfermero. En el análisis nosotros identificamos la contribución de los Directorios Curriculares, además de las estrategias que facilitan la enseñanza del cuidado humanizado. Para el desarrollo de esa competencia es fundamental énfasis en esa dimensión en la formación académica y la educación permanente.

Descriptores: Educación en enfermería; Estudiantes de enfermería; Humanización de la asistencia; Recursos humanos en salud.

*Trabalho vinculado ao Núcleo de Estudos e Pesquisas em Saúde Integral da Faculdade de Enfermagem da Universidade Federal de Goiás (NEPSI/FEN/UFG). 


\section{INTRODUÇÃO}

A humanização da assistência em saúde tem sido alvo de várias discussões Que sinalizam Que, humanizá-la não se resume na prestação de atendimento em local confortável, agradável e com profissionais educados, mas Que proporcione resolutividade nas ações prestadas ${ }^{(1)}$.

Porém o envolvimento do profissional com a doença e com os procedimentos necessários em sua decorrência muitas vezes leva a priorização do problema em detrimento da pessoa Que apresenta o problema, o Que acarreta a despersonalização da pessoa cuidada ${ }^{(2-4)}$. Nesse sentido, o processo de humanização do atendimento em saúde envolve a Qualificação dos profissionais, Que começa ainda nas universidades, bem como no contexto da educação continuada ${ }^{(5-7)}$.

Porém, o ensino do cuidado não se dá apenas com base apenas na dimensão do cognitivo com fundamentos teóricos humanistas, mas se constrói no cotidiano das interações entre educadores e educandos, com desenvolvimento de comportamentos, habilidades e atitudes humanistas ${ }^{(8-14)}$.

Uma das maneiras de formação desse modelo humanista e crítico de ensino-aprendizagem é a busca do conhecimento através da pesquisa sobre a prática do ensino do cuidado humanizado, visando novas concepções e reflexões, proporcionando o aperfeiçoamento da teoria aplicada à prática do ensino ${ }^{(9,11-13,15)}$.

Considerando que o pleno funcionamento do Sistema Único de Saúde depende de uma atitude humanista Que promova o acolhimento, a integralidade, entre outros aspectos, é de fundamental importância Que as escolas de Enfermagem viabilizem experiências de ensino Qualifieuem o cuidado em todas suas dimensões $^{(9,10,16)}$.

Esse aspecto é também destacado nas diretrizes curriculares para os cursos de graduação em enfermagem Que tem balizado as reformas dos currículos em todo o país ${ }^{(10,13)}$. A compreensão e a implementação dessas novas diretrizes pelas instituições formadoras podem garantir a formação de profissionais mais comprometidos com a Qualidade do cuidado integral, bem como devem contribuir com o desenvolvimento de profissionais dos serviços, Que também carecem compreender e desenvolver habilidades para uma atitude humanista $^{(1,2,8,10-12,17-19)}$.

Desta forma, as experiências dos alunos nas situações de aprendizado e sua convivência com os docentes possuem um papel de fundamental importância de como os comportamentos de cuidado humanizado serão transmitidos. "É necessário que o corpo docente identifieue o cuidado como um valor, reconhecendo e explorando seus significados"(2,13,20).

Considerando Que desde o inicio do movimento de reconstrução das diretrizes curriculares para os cursos de graduação em enfermagem ${ }^{(21)}$, bem como do lançamento do projeto nacional Humanização ${ }^{(22)}$ a Questão da humanização da assistência tem sido temática de interesse de pesquisadores, propusemos esse trabalho com objetivo de analisar a produção cientifica em periódicos nacionais sobre ensino do cuidado humanizado na graduação em enfermagem entre 1990/2008.

A decisão em focar a produção brasileira se justifica por se tratar de tema emergente, constituindo-se em preocupação atual da área da saúde, como forma de garantir os princípios do SUS (Sistema Único de Saúde) como universalização, descentralização, equidade e integralidade nos serviços de saúde.

\section{METODOLOGIA}

Estudo descritivo e exploratório, de natureza bibliográfica cujo propósito é exame de todo material escrito e publicado para levantamento e análise do Que se produziu sobre determinado assunto caracterizado como tema de pesquisa. A pesquisa bibliográfica permite ao pesQuisador contato direto com a produção sobre determinado assunto, permitindo maior cobertura dos fenômenos pesquisados ${ }^{(23)}$.

O período delimitado para a investigação foi entre 1990 a 2008 , justificado por resultados de estudo anterior ${ }^{(18)}$ Que sinaliza Que a humanização emerge como tema de interesse a partir da década de 90. Esse momento histórico coincide com a implantação de políticas de saúde cujo foco era garantir a humanização do atendimento em saúde no Brasil ${ }^{(22)}$.

A seleção do material pesquisado foi feita por meio de busca ativa em periódicos de enfermagem, considerando como critérios de inclusão sua indexação, regularidade, periodicidade, permitindo assim uma análise ao longo do período de estudo, Que estivessem disponíveis na BVS ou em outro site online.

Os periódicos Que atenderam os critérios de inclusão foram: Revista Brasileira de Enfermagem; Revista da Escola de Enfermagem da USP; Revista Gaúcha de Enfermagem; Acta Paulista de Enfermagem; Texto \& Contexto Enfermagem; Revista LatinoAmericana de Enfermagem; Revista de Enfermagem da UERJ; Cogitare Enfermagem; Revista da Escola de Enfermagem Anna Nery, Revista Eletrônica de Enfermagem, Revista Ciência Cuidado e Saúde, Revista Mineira de Enfermagem.

Para seleção dos artigos foi feita busca a partir de leitura prévia de todos os títulos, resumos e descritores, Que indicassem o ensino do cuidado humanizado. A seleção inicial resultou em 79 artigos Que foram lidos na íntegra, compilados, separados por periódicos, ano de publicação e volume. Após esse processo, apenas 23 artigos foram incluídos definitivamente para estudo, por tratarem exatamente do foco pretendido pela investigação.

O próximo passo consistiu da análise de cada artigo mediante um protocolo Que nos permitiu identificar dados sobre a distribuição dos artigos por ano ao longo do período estudado, procedência dos autores, natureza dos artigos, Que foram tratados por procedimentos estatísticos simples.

O conteúdo dos artigos foi submetido a uma análise Qualitativa, Que corresponde a procedimento mais intuitivo, maleável e adaptável a índices não previstos ${ }^{(24)}$. Para esse processo três peseuisadores por meio de consenso agruparam por semelhança de temas e enfoques, estabelecendo as seguintes categorias: A contribuição das Diretrizes Curriculares para Curso de Graduação em Enfermagem na formação do enfermeiro; Ensinando o Cuidado Humanizado; Metodologias de Ensino que favorecem o Cuidado Humanizado; A importância da experiência assistencial para a aprendizagem do Cuidado Humanizado.

\section{RESULTADOS E DISCUSSÃO}

A análise feita nos artigos vinte e três selecionados nos permitiu estabelecer um panorama geral sobre a publicação ao longo dos 


\begin{tabular}{|c|c|c|c|c|}
\hline Periódico & Ano & Autores & Procedência do $1^{\circ}$ autor & Tipo de artigo \\
\hline Rev Bras Enferm & 2000 & Santos et al & $\mathrm{RJ}$ & Pesquisa \\
\hline Rev Bras Enferm & 2000 & Esperidião e Munari & $\mathrm{GO}$ & Ensaio \\
\hline Rev Bras Enferm & 2004 & Motta & SC & Relato de Experiência \\
\hline Rev Esc Enferm USP & 2004 & Esperidião e Munari & $\mathrm{GO}$ & Pesquisa \\
\hline Rev Ciênc Cuidado e Saúde & 2007 & Lima et al & $\mathrm{GO}$ & Pesquisa \\
\hline Rev Esc Enferm USP & 2005 & Kantorski et al & RS & Pesquisa \\
\hline Rev Gaúcha Enferm & 1992 & Waldow & RS & Reflexão Teórica \\
\hline Rev Gaúcha Enferm & 1993 & Waldow & RS & Reflexão Teórica \\
\hline Rev Gaúcha Enferm & 2001 & Deves e Nunes & RS & Pesquisa \\
\hline Acta Paul Enferm & 2005 & Amorim e Oliveira & SP & Pesquisa \\
\hline Text e Contex Enferm & 2000 & Loureiro e Vaz & RS & Reflexão Teórica \\
\hline Rev Latino-am Enferm & 1993 & Freitas et al & SP & Reflexão Teórica \\
\hline Rev Latino-am Enferm & 1996 & Silva e Júnior & SP & Relato de Experiência \\
\hline Rev Latino-am Enferm & 1997 & Carvalho et al & SP & Pesquisa \\
\hline Rev Latino-am Enferm & 1998 & Jesus e Cunha & MG & PesQuisa \\
\hline Rev Latino-am Enferm & 2001 & Camacho e Santos & RJ & Reflexão Teórica \\
\hline Rev Latino-am Enferm & 2002 & Esperidião et al & $\mathrm{GO}$ & Pesquisa \\
\hline Rev Enferm UERJ & 2001 & Waldow & RS & Atualização \\
\hline Rev Enferm UERJ & 2001 & Figueiredo et al & $\mathrm{RJ}$ & Pesquisa \\
\hline Rev Enferm UERJ & 2003 & Shiratori & $\mathrm{RJ}$ & Reflexão Teórica \\
\hline Rev Enferm UERJ & 2005 & Ribeiro et al & $\overline{D F}$ & Reflexão Teórica \\
\hline Cogitare Enfermagem & 2002 & Paschoal & PR & Reflexão Teórica \\
\hline Cogitare Enfermagem & 2004 & Kletemberg & $\mathrm{PR}$ & Reflexão Teórica \\
\hline
\end{tabular}

\section{Quadro 1. Artigos estudados segundo periódico, ano de publicação, autores, procedência do primeiro autor e tipo de} artigo no período de 1990 a 2008.

anos, conforme pode ser observado no Quadro I.

Os dados da tabela mostram Que apenas as Revista da Escola de Enfermagem Anna Nery, Revista Eletrônica de Enfermagem e Revista Mineira de Enfermagem não publicaram artigos sobre o tema. A Revista Latino-Americana de Enfermagem foi a Que publicou o maior número de artigos $(06=26 \%)$, seguida da Revista de Enfermagem da UERJ $(04=18 \%)$, Revista Brasileira de Enfermagem $(03=13 \%)$, Revista Gaúcha de Enfermagem $(03=13 \%)$.

O maior número de trabalhos são pesquisas originais ( $10=44 \%)$, reflexões teóricas $(09=39 \%)$, seguidos de relato de experiência $(02=9 \%)$, ensaio $(0 \mathrm{l}=4 \%)$, e atualização $(0 \mathrm{l}=4 \%)$.

$\mathrm{O}$ agrupamento dos artigos por semelhança nos permitiu constatar sua relação com as mudanças curriculares, metodologias de ensino e experiências assistenciais acerca do enfoque do cuidado humanizado na definição das metas do projeto político pedagógico dos cursos de graduação, Que será tratado a seguir.

\section{A contribuição das Diretrizes Curriculares para Curso de Graduação em Enfermagem na formação do enfermeiro}

Essa categoria reúne cinco estudos (22\%) Que tratam do processo de construção das diretrizes curriculares e algumas diferenças Que ocorreram após Resolução CNE/CES de 2001. Fundamentada no modelo ético-humanista, repercute no processo de formação dos enfermeiros.

Desde 1993 já se discutia uma reforma curricular Que despertasse reflexão crítica acerca do homem, da sociedade, da saúde e do processo saúde-doença. Artigo Que explora essa temática, oriundo de docentes da Comissão de Reestruturação
Curricular da Escola de Enfermagem de Ribeirão Preto/USP, propunha a formação do enfermeiro generalista, através de abordagem multidisciplinar, embasada em princípios filosóficos Que enriquecessem e fortalecessem a formação profissional(25).

Assim a reestruturação era proposta para avançar além do modelo biomédico até então vigente, introduzindo referenciais de um paradigma na perspectiva holística para formação profissional e Que influenciasse o campo da prática, favorecendo uma assistência humanizada ${ }^{(26)}$.

Nesse contexto de mudanças, o projeto pedagógico do curso das instituições formadoras deve contemplar o desenvolvimento global, harmônico, holístico, da pessoa como unidade cognitiva e emocional $^{(10,13,14)}$. Nesse sentido, as diretrizes curriculares para os cursos de graduação em enfermagem valorizam, principalmente, o modelo ético-humanista no Qual ela foi embasada. As orientações deste documento deixam "clara a preocupação com a solidariedade e cidadania, como saber conviver, com o aprender a ser e o aprender a viver juntos, elementos Que constituem a essência do humanismo e da ética como mola mestra do comportamento humano e das ações profissionais" ${ }^{(10)}$.

Neste sentido, a implementação dessa nova diretriz apresenta condições favoráveis para Que os acadêmicos de enfermagem tenham uma formação humanista de fato, e Que isso possa refletir Que a assistência à saúde seja humanizada ${ }^{(10)}$ conforme hoje preconiza o Sistema Único de Saúde ${ }^{(1,3)}$.

\section{Ensinando o Cuidado Humanizado}

Nessa categoria foram incluídos nove artigos (39\%), Que relatam e discutem o significado do cuidado/cuidar no ensino de 
enfermagem, salientando aspectos políticos, éticos, morais e filosóficos Que permeiam um currículo direcionado para o cuidado na enfermagem.

O conteúdo desses artigos sinaliza Que ensinar o cuidado humanizado é desenvolver o sentido de Ser humano no contexto globalizado, mostrando Que o aluno deve ser ético sem impor seu poder simbólico no cuidar, apoiado em uma formação críticoreflexiva ${ }^{(6)}$. Para ensinar o cuidado humanizado o corpo docente também precisa humanizar suas relações e investir no "desenvolvimento da abordagem holística no processo de formação do enfermeiro, considerando a humanização do ensino uma estratégia necessária para o estabelecimento de relações humanizadas com as pessoas Que este profissional assiste"(II).

Além de conhecer os significados e valores do ensinar e aprender o cuidado/cuidar é fundamental o desenvolvimento do processo saber e fazer Enfermagem focado em "estratégias de ensinar e aprender cuidado, com pontos de convergência que caminhem para a integração"(12). Esse é um ponto complexo discutidos por vários autores, haja vista Que exige disposição para mudança do corpo docente, formado sob a égide de uma educação tradicional ${ }^{(1 .}$ 2.4.6.9, 12-14). Nesse contexto, a mudança nas tecnologias de ensino se mostra como ferramenta indispensável para o avanço nessa Questão $^{(2,6,9,12-15)}$.

\section{Metodologias de Ensino Que favorecem o Cuidado Humanizado}

Nesta categoria foram incluídos cinco artigos (22\%) Que abordam metodologias que facilitam o ensino humanizado focado na interação humana Que trazem resultados satisfatórios e também se mostram eficientes instrumentos do cuidado.

Os referidos estudos destacam a comunicação ${ }^{(26)}$, a valorização do desenvolvimento dos órgãos do sentido ${ }^{(6)}$ como apoio para a expressão do cuidar. Esse aprendizado "envolve transversalidade, escuta e muito exercício para perceber sentir é compreender e envolve afetividade, imaginação, intermediação, percepção e escuta"(27). As metodologias utilizadas nos estudos buscam uma prática embasada na compreensão holística do ser humano.

\section{A importância da experiência assistencial para a aprendizagem do Cuidado Humanizado}

Esta categoria reúne Quatro estudos (17\%) Que evidenciam como a experiência assistencial é essencial para orientar o aprendizado do cuidado humano. Nesse contexto, o papel do professor se constrói no desafio de ensinar a cuidar focado principalmente, nas interações estabelecidas entre os atores do processo (docentes, clientes assistidos e a equipe de saúde), sendo um modelo para o aluno.

Assim, durante as atividades práticas, os alunos têm a oportunidade de fazer-aprender o cuidado de forma sensível, tendo oportunidade de articular as dimensões cognitivas, emocionais, comportamentais e atitudinais do conhecimento ${ }^{(13,15)}$.

Ao experimentar o cuidado por meio das interações com o outro valorizam a dimensão ética e humana do cuidar, aprendem a enfrentar as dificuldades e se tornam profissionais maduros e capacitados para o cuidado humano(13). É no contexto da prática também Que os alunos se desenvolvem enquanto pessoas, o Que os torna mais fortalecidos para enfrentar a complexidade da vida profissional ${ }^{(2)}$.

As experiências adQuiridas na prática profissional são importantes para a formação do acadêmico, por proporcionar a troca de experiências e pela vivência, propiciando o surgimento de novas idéias, novos conceitos, novas direções Que contribuem com o aprendizado.

\section{CONSIDERAÇÕES FINAIS}

A realização desse estudo nos permitiu conhecer o panorama nacional sobre a situação, tendências do conhecimento e experiências, produzidas pelos enfermeiros brasileiros sobre o ensino do cuidado humanizado dispensado aos alunos de graduação em enfermagem, no período de 1990 a 2008.

Esse conhecimento sinaliza aos cursos de graduação em Enfermagem a importância de investimento nessa direção para a implementação dos novos projetos pedagógicos dos cursos de graduação.

As características desse ensino sinalizam Que este deve estar focado na articulação teórico-prática de aprender/fazer o cuidado de enfermagem e Que, a interação do professor nesse campo com os diversos atores que nele interagem são a base do processo ensino-aprendizagem.

Verificamos Que há expansão dessa produção, Que coincide com implantação do HUMANIZASUS e das novas diretrizes curriculares, sendo que entre 2000 e 2003 ocorreu o maior número de publicações.

Quando nos dedicamos ao estudo da temática não ignoramos o valor dos aspectos técnicos Que envolvem a formação dos profissionais de saúde. Articulados aos aspectos relacionais Qualificam a assistência e valorizam as ações dos profissionais. Na realidade, com os desafios postos as instituições formadoras para a mudança de paradigma no ensino, é fundamental tornar o aluno um sujeito mais ativo e critico no seu processo de aprendizado, para Que este possa, eneuanto profissional, mudar a realidade da assistência no Brasil. Estamos, pois, diante de um caminho sem volta.

As tendências Que se colocam nessa perspectiva mostram Que não é possível hoje, planejar QualQuer ação de formação de recursos humanos em saúde, Quer seja no contexto da acadêmica ou nos serviços e comunidade, desarticulada dos pressupostos do SUS, da valorização da participação popular nesse processo e da importância de estimular os profissionais para uma atuação comprometida, criativa, critica e reflexiva.

Acreditamos que um dos caminhos para essa conquista esteja na formação acadêmica Que, ao apresentar os fundamentos do cuidado humano ao aluno na graduação, contribui para uma atuação diferenciada no campo de trabalho. Por sua vez, a implementação do SUS exige profissionais com essa competência, o que sinaliza a importância da educação permanente, no estabelecimento de uma conduta mais humanista e integradora na prática profissional. 


\section{REFERÊNCIAS}

I. Deslandes SF. Análise do discurso oficial sobre a humanização da assistência hospitalar. Ciênc Saúde Coletiva 2004; 9(1): 7-I4.

2. Esperidião $\mathrm{E}$, Munari DB. A formação integral dos profissionais de saúde: possibilidades para a humanização da assistência. Cienc Cuid Saúde 2005; 4(2): 163-70.

3. Santos-Filho SB. Perspectivas da avaliação na Politica Nacional de Humanização em Saúde: aspectos conceituais e metodológicos. Ciênc Saúde Coletiva 2007; 12(4): 999-1010.

4. Arone EM, Cunha ICKO. Tecnologia e humanização: desafios gerenciados pelo enfermeiro em prol da integralidade da assistência. Rev Bras Enferm 2007; 60(6): 72 l-3.

5. Oliveira SG. Humanização da assistência: um estudo de caso. Rev Adm Saúde 2007; 9(35): 55-62.

6. Shiratori K, Figueiredo NMA, Porto F, Silva CSI, Teixeira MS. O sentido de Ser Humano: uma base reflexiva para o cuidado de enfermagem. Rev Enferm UERJ 2003; I I (2): 2 I 2-6.

7. Santos VMG. Humanização nos serviços: experiência voltada à educação permanente. Rev Baiana Saúde Pública 2007; 3 I (supl. I): 54-60.

8. Rivero DE, Erdmann AL. The power of loving humane care in nursing. Rev Latino-Am Enfermagem 2007; 15(4): 61 8-25.

9. Damas KCA, Munari DB, Siqueira KM. Cuidando do cuidador: reflexões sobre o aprendizado dessa habilidade. Rev Eletrônica Enferm 2004; 6(2): I-5.

10. Ribeiro IP, Tavares M, Esperidião E, Munari DB. Análise das diretrizes curriculares: uma visão humanista na formação do enfermeiro. Rev Enferm UERJ 2005; 13(3): 403-9.

11. Motta MG. Cuidado humanizado no ensino de enfermagem. Rev Bras Enferm 2004; 57(6): 758-60.

12. Amorim RC, Oliveira EM. O ensino e práticas de cuidado: o caso de um curso de graduação em enfermagem. Acta Paulista Enferm 2005; 18(I): 25-30.

13. Lima JOR, Esperidião E, Munari DB, Souza JC. Aprendendo o cuidado humanizado: perspectiva do graduando de enfermagem. Rev Ciênc Cuid Saúde 2007; 6(1): I I-20.

14. Backes DS, Lunardi-Filho WD, Lunardi VL. A construção de um processo interdisciplinar de humanização à luz de Freire. Texto Contexto Enferm 2005; 14(3): 427-34.

15. Casate IC, Corrêa AK. Vivências de alunos de enfermagem em estágio hospitalar: subsídios para refletir sobre a humanização em saúde. Rev Esc Enferm USP 2006; 40(3): 32 I-8.

16. Simões ALA, Rodrigues FR, Tavares DMS, Rodrigues LR. Humanização na saúde: enfoQue na atenção primária. Texto Contexto Enferm 2007; 16(3): 439-44.

17. Beck CLC, Gonzáles RMB, Denardin IM, Trindade LL, Lautert L. A humanização na perspectiva dos trabalhadores de enfermagem. Texto Contexto Enferm 2007; 16(3): 503-10.

18. Casate JC, Corrêa AK. Humanização do atendimento em saúde: conhecimento veiculado na literatura brasileira de enfermagem. Rev Latino-Am Enfermagem 2005; 13(1): 105-11.

19. Amestoy SC, Schuwartz E, Thofehrn MB. A humanização do trabalho para os profissionais de enfermagem. Acta Paulista Enferm 2006; 19(4): 444-9.

20. Waldow VR. Cuidado humano: o resgate necessário. $3^{\text {a }}$ ed. Porto Alegre: Sagra Luzatto; 2001.

21. Ministério da Educação e Cultura (BR). Conselho Nacional de Educação. Resolução CNE No 3 - Diretrizes Curriculares Nacionais do Curso de Graduação em Enfermagem. Brasília: Ministério da Educação e Cultura; 2001 .

22. Ministério da Saúde (BR). Secretária Executiva. Núcleo Técnico da Politica Nacional de Humanização. HumanizaSUS: política nacional de humanização [internet]. Brasília: Ministério da Saúde; 2003.

23. Ruiz |A. Metodologia Científica. $5^{\text {a }}$ ed. São Paulo: Editora Atlas; 2002.

24. Bardin L. Análise de Conteúdo. Lisboa: Edições 70; 1977.

25. Freitas DMV, Santos BMO, Vietta EP, Gomes ELR, Valle ERM, Mendes IJM, et al. Reforma Curricular de Graduação en Enfermagem - Escola de enfermagem de Ribeirão Preto Universidade de São Paulo. Comissão de Reestruturação Curricular da Escola de Enfermagem de Ribeirão Preto-USP. Rev Latino-Am Enfermagem 1993; 2(1): 35-5I.

26. Carvalho EC, Bacchion MM, Almeida LCP, Medeiros RN. O ensino de comunicação de enfermagem: um desafio. Rev LatinoAm Enfermagem 1997; 5(3): 27-34.

27. Figueiredo NMA, Porto IS, Santos I, Cardoso FCA, Souza FS. Ensinando alunas(os) a sentir: areuitetando uma semiologia da expressão para cuidar - os sentidos e o cuidado. Rev Enferm UERI 200 I; 9(3): 217-24. 